\title{
Correction to: The hierarchy of different treatments for myogenous temporomandibular disorders: a systematic review and network meta-analysis of randomized clinical trials
}

\author{
Essam Ahmed Al-Moraissi ${ }^{1}$. Paulo César Rodrigues Conti ${ }^{2} \cdot$ Abdulmalik Alyahya $^{3} \cdot$ Khaled Alkebsi $^{4}$. \\ Ahmed Elsharkawy ${ }^{5} \cdot$ Nikolaos Christidis $^{6}$
}

Published online: 5 November 2021

(c) The Author(s) 2021

\section{Correction to: Oral and Maxillofacial Surgery (2021) https://doi.org/10.1007/s10006-021-01009-y}

Originally, the article has been published online with an error in affiliations. This has been correctly presented here.

The original article has been corrected.

Publisher's note Springer Nature remains neutral with regard to jurisdictional claims in published maps and institutional affiliations.

The original article can be found online at https://doi.org/10.1007/ s10006-021-01009-y.

Essam Ahmed Al-Moraissi

dressamalmoraissi@gmail.com;

dr_essamalmoraissi@yahoo.com

1 Department of Oral and Maxillofacial Surgery, Faculty of Dentistry, Thamar University, Thamar, Yemen

2 Department of Prosthodontics, Bauru School of Dentistry, University of São Paulo, São Paulo, Brazil

3 Oral and Maxillofacial Surgery Department, King Abdulaziz Medical City - National Guard, Riyadh, Saudi Arabia

4 Department of Oral and Maxillofacial Surgery, West China Hospital of Stomatology, Sichuan University, Chengdu, Sichuan, China

5 Department of Oral and Maxillofacial Surgery, Cairo Faculty of Dentistry, Cairo University, Cairo, Egypt

6 Division of Oral Diagnostics and Rehabilitation, Department of Dental Medicine, Karolinska Institutet, and Scandinavian Center for Orofacial Neurosciences, SE-141 04 Huddinge, Sweden 